\title{
Féeries
}

Études sur le conte merveilleuX, XVII -XIX siècle

Contes et morale(s)

\section{Écrire dans la gueule du loup}

Writing in the Woolf's Mouth

\section{Hélène Merlin-Kajman}

\section{(2) OpenEdition}

Journals

Édition électronique

URL : http://journals.openedition.org/feeries/994

ISSN : 1957-7753

Éditeur

UGA Éditions/Université Grenoble Alpes

Édition imprimée

Date de publication : 15 octobre 2016

Pagination : 27-45

ISBN : 978-2-8310-335-3

ISSN : 1766-2842

Référence électronique

Hélène Merlin-Kajman, «Écrire dans la gueule du loup », Féeries [En ligne], 13 | 2016, mis en ligne le 01 janvier 2017, consulté le 08 septembre 2020. URL : http://journals.openedition.org/feeries/994

(c) Féeries 


\section{ÉCRIRE DANS LA GUEULE DU LOUP}

$E$ $N$ ÉCRIVANT, en juin 20II, trois fables pour la rubrique de création du site de "Transitions ${ }^{\mathrm{I}}$ », je ne savais pas que j'allais y prendre goût et même m'y enhardir. Mes raisons de départ pour le faire étaient très circonscrites et quasiment expérimentales : je voulais ouvrir un chemin, inviter d'autres à me suivre dans cette relance d'un genre mineur, mais rendu majeur et même inimitable par La Fontaine (d'où la hardiesse).

Le mouvement "Transitions» avait été créé un an auparavant. Son manifeste incitait à «transiter, transhumer, traditionner, transporter» :

L'âpreté de l'histoire ne condamne pas au reniement de tout passé. Pour peindre l'avenir, il faut couleurs et pinceaux, il faut géométrie, science des signes, des choses humbles, des choses savantes. Il faut, plutôt que continuer à faire le compte hébété des cicatrices, se souvenir des gestes, de la main qui dessine, du regard émerveillé qui se pose. Que suintent les plaies, hélas, il est trop vrai — et transit l'imagination : mais transir l'imagination ne les guérit pas².

S’appuyant sur la distinction entre récit d'expérience et information établie par Walter Benjamin dans son texte célèbre "Le Conteur ${ }^{3}$ ", le manifeste concluait sur le privilège que le mouvement accorderait aux «histoires» :

Que ce lieu soit de bon conseil et de bon partage. Racontons-nous donc des histoires, des expériences.

En somme, la nécessité de réinvestir la fable, littérairement parlant, était ici inscrite en creux.

J'avais des raisons plus particulières de désirer tenter l'expérience. Cela faisait bien vingt ans que je lisais assidûment des albums de jeunesse. J'avais commencé avec mes enfants, puis continué sur la lancée dans le cadre

I. < http://www.mouvement-transitions.fr/juste/presentation.html>

2. < http://www.mouvement-transitions.fr/manifeste.html>

3. W. Benjamin, «Le Conteur», dans Euvres [1972], t. III, trad. M. de Gandillac, R. Rochlitz et P. Rusch, Paris, Gallimard, coll. «Folio essais», 2000. 
d'une réflexion portant sur l'enseignement et l'éducation. Ce qui m'avait rapidement frappée, c'était la récurrence du détournement des valeurs morales traditionnellement accrochées aux figures animales ${ }^{4}$. Pour ne prendre qu'un exemple, on ne compte plus les loups gentils ou vertueux dans ces ouvrages. Dans Docteur Loup d'Olga Lecaye par exemple (I994), album composé d'un texte sur la page de gauche et d'une image sur la page de droite, tout commence par la maladie d'un «Petit Lapin», figure animale évidemment choisie en raison de l'hypocoristique si usuel pour appeler un enfant de façon affectueuse : «Sa maman décide de l'emmener voir un docteur, car elle a peur pour son Petit Lapin's.» Suit une errance de médecin en médecin : la taupe lui prescrit de creuser des tunnels, le chien, de manger des os, l'oiseau, de voler, etc. Ces divers animaux sont donc des figures allégoriques de l'amour-propre. Le docteur Chat, par exemple, recommande au petit lapin de manger «un bon pâté de rat ou une tarte à la souris». Conformément à la tradition allégorique des fables, la fonction symbolique de la figure animale s'adosse à ses déterminations "naturelles» : même si le message est légèrement déplacé par rapport à cette tradition puisque les deux protagonistes principaux du conte, maman Lapin et son fils, ne sont pas des proies pour le chat, une menace plane autour de ces figures de médecins. La logique est sérielle : face à la détresse de la mère lapin, toutes les espèces se valent dans leur cécité égoïste, leur enfermement dans leur intérêt particulier. Sur l'image, ils sont du reste très peu anthropomorphiques, voire pas du tout, contrairement aux deux lapins qui ont un corps, des allures, des gestes et des vêtements humains, comme bientôt le loup.

Car tout va changer avec Docteur Loup.

C'est un hibou qui conseille à la mère désespérée de se rendre chez lui. Maman Lapin s'affole puisque «les loups sont méchants» et «mangent les petits lapins» : vérité naturelle et sens symbolique se superposent ici, et la mère lapin fait état d'un savoir qui pourrait être celui de l'instinct mais qui, bien sûr, puisqu'elle parle, est celui de la culture et de la tradition allégorique, cette dernière elle-même fondée sur l'observation, l'expérience, et l'inférence morale.

La nuit est en train de tomber, l'image de la forêt est effrayante. Sans attendre la réponse de la mère lapin, le hibou hulule pour appeler Docteur Loup qui apparaît aussitôt. Mais «apparaît» n'est pas totalement exact, car l'illustration participe activement au suspense : occupant tout le premier

4. Voir mon livre La Langue est-elle fasciste? Langue, pouvoir, enseignement, Paris, Seuil, 2003.

5. O. Lecaye, Docteur Loup, Paris, École des loisirs, 1994, p. 4. 
plan de l'image, mais de dos, la silhouette énorme du loup revêtu d'un grand manteau, d'un chapeau recouvrant complètement sa tête est donc invisible au lecteur qui ne voit de lui qu'une main tenant la crosse d'une canne. Les deux lapins sont au contraire situés à la fois face au loup et face au lecteur, qui reçoit en plein visage l'effroi de l'enfant lapin et de sa mère, placés au deuxième plan, entre deux ombres d'arbre menaçantes. Suit un court dialogue :

"Je vous en supplie, Docteur Loup, ne mangez pas mon fils!»

"Ha ha ha!» Le loup éclate de rire. "Qu'en dis-tu mon garçon? Tu crois que j'ai envie de te manger?"

«N... non.» Marc a très peur. Mais il ne se sauve pas. Il voudrait bien savoir à quoi cela ressemble, un loup, de près.

L'illustration suivante ne montre pas davantage le loup, seulement le bas de son corps, situé cette fois au second plan, dans le coin droit en haut de la page : on aperçoit le manteau tombant bas sur ses jambes et, un peu devant elles, le bas de la canne. Le reste du loup est hors champ. Cette quasi-invisibilité est d'autant plus menaçante que le petit lapin et sa mère, placés quant à eux au premier plan, se trouvent cette fois enveloppés par l'ombre du loup. Plus précisément, placé entre le loup et sa mère, le petit lapin invite cette dernière à suivre le loup. Mais elle prend peur, et l'illustration suivante la montre se cachant les yeux et commençant à fuir tandis que l'enfant, lui, est entraîné par le loup dont on ne voit toujours pas le visage (seulement sa patte tenant la main du petit lapin) : "Sauvonsnous, Marc!" crie-t-elle en courant vers les arbres."

Vient alors une image saisissante : celle de la mère lapin courant de façon panique, seule, au milieu des troncs d'arbres sombres. Sans doute a-t-elle la tête tournée vers l'arrière : c'est que, dit le texte, après avoir couru "bien cinq minutes sans s'arrêter", elle se retourne pour s'apercevoir qu'il ne l'a pas suivie. Mais elle a commencé par partir comme une flèche sans son "Petit Lapin", c'est-à-dire d'abord sans se retourner : ce bref moment, l'image ne le montre pas, elle se contente de superposer la fuite et le retournement.

Retournant sur ses pas, elle surmonte alors sa peur :

Très loin, elle aperçoit une lueur. Cela doit être la maison du Docteur Loup. Malgré sa peur, maman Lapin décide d'y aller.

On devine la suite. La mère lapin (et le lecteur) découvre le petit lapin installé sur les genoux du loup en train de lui raconter une histoire. Son "visage», cette fois révélé par l'illustration, est souriant et chaleureux. Et il donne son diagnostic, un diagnostic rassurant sur la maladie du petit lapin. 
Quelle est la morale explicite de la fable? La peur n'est pas seulement mauvaise conseillère : elle n'a pas d'objet. Il ne faut se fier ni aux apparences, ni aux rumeurs, ni à la culture, ni aux lois naturelles. La curiosité favorable à l'égard de l'autre, aussi inquiétant qu'il puisse être, est mieux fondée que la peur. À ceci s'ajoute une morale implicite: mieux vaut suivre un inconnu que sa mère; l'autre dont on se méfie le plus se révèle (toujours?) le meilleur; même, il protège mieux que la mère, défaillante. Celle-ci est aliénée à un savoir culturel qui (la) fourvoie. La doxa dit que les loups sont méchants et mangent les petits lapins : mais ce n'est que doxa, et la mère trop crédule constitue pour son fils un soutien fragile et mal orienté. Le soin (le salut) du petit lapin va provenir de la rencontre totalement imprévisible, hasardeuse, périlleuse du point de vue de la doxa, avec l'autre étranger.

Pourquoi ce retournement de la valeur allégorique traditionnelle du loup, et du loup seul? On le comprend sans peine : le loup «bon» soutient un message antiraciste et multiculturaliste. Il figure d'autant plus facilement l'étranger ethnique que la tradition allégorique l'accole généralement à l'agneau : la polarité symbolique du noir et du blanc a ajouté ses propres connotations et équivalences à la valeur négative du loup, favorisant ainsi des projections occidentalocentriques racistes. Renverser la valeur morale du loup, c'est combattre ce substrat culturel. Pour être plus précis, c'est au niveau des affects que se renverse la tradition allégorique, car ce sont les affects eux-mêmes qui sont symboliquement investis : l'effroi qui depuis toujours est associé au loup devient ici la figure allégorique de l'effroi injustement entretenu par les discours xénophobes.

Or, cette opération de retournement généreux, qui veut évidemment éduquer à la tolérance, a un coût : l'étranger reste figuré par un loup, et, en ce sens, le discours ethnocentriste se trouve reconduit. Car, dans la tradition allégorique, la valeur symbolique de la figure animale n'est pas arbitraire, mais motivée : comme dans le cas du chat et de la souris, elle repose sur la vérité d'un rapport de force inscrit dans la nature - le loup est bel et bien un prédateur pour l'agneau ou pour le lapin. La nouvelle morale repose donc sur une sorte de déni ou de refoulement problématiques : le déni des "appétits», des rapports de force et, finalement, de l'expérience. Et ce refoulement maintient — dans le non-dit — la connotation de menace dont est enveloppée la relation à l'autre étranger, tout en interdisant qu'un sentiment de menace puisse fonctionner comme un indice parfois fiable.

Certes, les fables de La Fontaine nous ont préparés à ces réévaluations allégoriques. «Le Loup et les Bergers» $(\mathrm{X}, 5)$ par exemple s'ouvre sur un vers qui, avec un humour souligné, lance une bombe dans la tradition cultu- 
relle : «Un loup rempli d'humanité / (S'il en est de tels dans le monde) / Fit un jour sur sa cruauté, / Quoiqu'il ne l'exerçât que par nécessité, / Une réflexion profonde. " En quelques vers, La Fontaine nous met en alerte et nous demande, à nous aussi, de réfléchir. Le loup prouve immédiatement son humanité en raisonnant avec exactitude sur son propre cas, c'est-à-dire sur le paradoxe de sa "cruauté». Nécessité interprétée comme un vice moral, le caractère carnassier du loup est ainsi situé à mi-chemin de la loi naturelle (animale) et de la loi culturelle (humaine). En ce sens, sa "cruauté» ne lui appartient pas, elle est un produit symbolique d'où résulte la sentence judiciaire le condamnant universellement : " Je suis haï, dit-il, et de qui ? De chacun. / Le loup est l'ennemi commun."

Pourtant, à y regarder de plus près, la vérité naturelle (l'ordre de la nécessité) devrait conduire les hommes à réévaluer à la baisse l'allégorisation morale dont le loup fait l'objet :

Il n'est marmot osant crier

Que du loup aussitôt sa mère ne menace.

Le tout pour un âne rogneux,

Pour un mouton pourri, pour quelque chien hargneux,

Dont j'aurai passé mon envie.

La réalité carnassière du loup est "objectivement» (du point de vue de la factualité de la «nature») très en deçà de ce dont il est la figure exagérée tant du point de vue qualitatif (les animaux qu'il mange sont soit déjà morts, soit de peu de qualité ${ }^{6}$, il n'est donc pas un danger si grand pour l'élevage), que quantitatif (le loup ne mange pas les enfants, il n'est pas un ennemi universel).

Cependant, puisque c'est un loup «rempli d'humanité», il commence par se soumettre au verdict de la loi (symbolique et judiciaire ${ }^{7}$ ), fut-ce problématique pour sa propre survie (pour la «nécessité» de sa propre nature) :

Eh bien! ne mangeons plus de chose ayant eu vie;

Paissons l'herbe, broutons, mourons de faim plutôt.

Est-ce une chose si cruelle?

Vaut-il mieux s'attirer la haine universelle?

6. Évidemment, on ne peut pas éliminer l'hypothèse que La Fontaine ironise sur les raisons du loup, qui fait ici un plaidoyer pro domo : le loup travestit peut-être la «réalité». Mais dans la diégèse, qui veut-il persuader? C'est à lui-même qu'il parle... Le ton est plus celui du constat amer que du discours sophistique. C'est donc le fabuliste qui adresse le raisonnement du loup aux lecteurs : il me paraît faire parler le loup pour défendre sa cause légitime.

7. La fable évoque notamment la situation anglaise : "On y mit notre tête à prix.» (v. II) 
Mais la fable va soudain bifurquer au moment où le loup rencontre des bergers qu'il voit en train de manger des moutons : de façon parfaitement fondée et cohérente, le spectacle lui fait opérer une révision allégorique de son propre cas et diriger l'accusation sur le cas des bergers, dont la fonction allégorique est ici mobilisée. Puisque ces derniers mangent ceux dont ils ont la garde (l'allégorie se politise : les bergers deviennent les figures du tyran, et les moutons, celles d'un peuple abusé), pourquoi le loup, qui a du moins pour lui la loi de la nécessité, devrait-il s'abstenir d'en faire autant? Parce que «[c]hiens, chasseurs, villageois, s'assemblent pour sa perte» (v. 8) ? Sans doute. Et c'est donc que, dans le vis-à-vis du loup et des bergers, la raison du plus fort se situe ici du côté de ces derniers, non du loup. Les bergers (et, à travers eux, la loi humaine) figurent le Pouvoir, dont la force est dissimulée par l'imaginaire de la Justice, comme l'a maintes fois montré Louis Marin. L'allégorie dénonce ici la tyrannie du pouvoir des allégories, l'hypocrisie de la morale — le mensonge de la culture, de la symbolisation.

L'affabulation humaine, sa manière de symboliser, est donc aussi ce que la fable lafontainienne met en question, ce qu'elle allégorise. Ce procès de la culture et de la symbolisation est encore plus net dans «L'Homme et la Couleuvre» $(\mathrm{X}, \mathrm{I})$, où la situation initiale motivant la rencontre et le différend des deux protagonistes n'a pas d'autre fondement que l'immédiate interprétation morale de l'animal par le regard humain :

Un homme vit une couleuvre.

«Ah! méchante, dit-il, je m'en vais faire une œuvre

Agréable à tout l'univers.» (v. I-3)

Dès qu'il aperçoit la couleuvre, l'homme opère une lecture allégorique qui la range sous le genre "serpent», figure du mal. C'est pourquoi le procès qui va se dérouler ne porte pas vraiment sur la culpabilité objective de la couleuvre, mais sur la légitimité de l'interpellation symbolique et sur le référent légitime du symbole : qui, de la couleuvre ou de l'homme, pourra être dit «animal pervers» et "symbole des ingrats»?

La couleuvre a en effet contesté immédiatement l'autorité ou la légitimité de l'homme, contrairement à l'agneau du «Loup et l'Agneau». Certes, c'est bien aussi «la raison du plus fort" qui va sceller son sort : mais le procès où auront défilé trois témoins (la vache, le bœuf et l'arbre), accusant à chaque fois l'homme d'être "le symbole des ingrats", aura "convaincu " l'homme (v. 79) : quand ce dernier tue la couleuvre, le geste, devenu un meurtre, a cessé de pouvoir se présenter comme l'application d'une sentence légitime. Au niveau de la fable, l'homme est devenu la figure allégorique d'un pouvoir arbitraire. 
On remarquera cependant que, dans "L'Homme et la Couleuvre», l'homme n'a pas faim, n'a pas d'appétit ni de désir autre que celui d'exercer sa puissance symbolique. La couleuvre et lui n'entrent pas dans un rapport de nécessité, mais dans une relation prescrite par la tradition culturelle qui a symbolisé le diable par le serpent. Même si c'est moins évident, il en va de même dans "Le Loup et les Bergers" puisque les moutons ne donnent pas vraiment lieu à une personnification, ni ne se trouvent à proprement parler au centre du drame : l'enjeu dramatique et moral de la fable se situe entre deux figures - le loup, l'homme - dont le conflit symbolique ne trouve qu'une motivation très indirecte dans la vérité naturelle de leur relation.

La configuration est assez différente dans "Le Loup et l'Agneau». Certes, le loup y symbolise l'arbitraire du pouvoir comme l'homme dans "L'Homme et la Couleuvre» et les bergers dans «Le Loup et les Bergers». Car s'il n'était qu'un loup, il n'aurait nul besoin de s'habiller de la justice pour satisfaire son appétit et dévorer l'agneau. Les derniers mots de la fable, "sans autre forme de procès", sont à lire ironiquement : quoique caricatural, il y a bel et bien eu procès. La fable représente, pour la condamner, une scène judiciaire expéditive, comme dans "L'Homme et la Couleuvre", à ceci près que l'agneau fait la bêtise de chercher à se justifier, acceptant ainsi les présupposés judiciaires imposés par le loup. Avec sa déférence et ses arguments, il incarne une crédulité idéologique propre à renforcer la pseudo-légitimité du loup, contrairement au parler-vrai de la couleuvre face à l'homme.

Toutefois, à un autre niveau d'analyse (celui de la motivation des symboles), c'est tout de même le rapport de force physique et la "nécessité» naturelle de l'appétit du loup qui expliquent que le loup dévore l'agneau. Aussi complexe que se présente la signification morale des fables de La Fontaine, jamais un loup n'y devient l'ami ou le bienfaiteur d'un agneau, jamais le chat ne noue amitié avec une souris, sauf par un pacte intéressé qui en général tourne mal pour le plus faible. Les fables respectent une sorte de principe de réalité incontournable. Certaines sont même chargées de le rappeler. Ainsi, la fable "Le Chat et les Deux Moineaux» (XII, 2) présente une situation initiale d'amitié entre un chat et un moineau. Là-dessus, arrive un autre moineau, qui joue avec le premier, se dispute avec lui. Le chat le croque, trouve la proie délicieuse - et croque son ami.

En fait, dans la tradition allégorique, certains animaux vont par paire : le loup et l'agneau ou le chat et la souris allégorisent des relations plus que des défauts ou des types séparés que le hasard de la vie ferait se rencontrer et interagir. D'où la puissance particulière du renard : le renard, c'est celui 
qui renverse les situations parce qu'il est mobile, hors relation, plastique - il ruse avec les codes et sait les retourner à son avantage.

Pour revenir à Docteur Loup, non seulement le conte dénie la "nécessité", mais ignore la signification allégorique de la relation elle-même. Le sens moral devient confus - et l'expérience (pour reprendre le terme investi par "Transitions» à la suite de Benjamin), inexistante.

Je viens de résumer les réflexions qui étaient les miennes lorsque je me suis lancée dans l'aventure des fables ${ }^{8}$. Mon premier projet n'était pas d'en inventer, mais d'en réactualiser de très connues pour en relancer l'usage -l'expérience. Je me suis très vite emparée du cas du loup en écrivant deux versions successives du "Loup et l'Agneau ". Mais il m'a en fait embarrassée. De cet embarras, j'ai choisi de faire l'objet de ma fable, en déléguant ce sentiment au conteur lui-même, sommé par l'enfant à qui il adresse sa fable, et qui connaît la version de La Fontaine, de "sauver l'Agneau". La dimension méta-allégorique présente dans «Le Loup et les Bergers» et "L'Homme et la Couleuvre» est ainsi confiée à la relation entre le conteur et l'enfant. Car mes fables sont aussi des mises en scène de fables. L'enfant initialement inventé ou imaginé par le "je» narrateur (qui ne devient "conteur» qu'en présence de celui-là) veut qu'on lui raconte des fables, mais sur un certain mode, ce que le conteur ne lui concède jamais facilement ni totalement. Ici par exemple, l'enfant lui suggère de mettre dans la tête de l'agneau un peu de la ruse du renard; à quoi le conteur objecte : "S’il avait une cervelle de renard, la fable s'appellerait "Le loup et le renard", voilà tout. Et tu sais bien que ça ne donnerait pas du tout la même fable...»

Comment sauver l'agneau? se demande donc le conteur. Bien sûr, j'aurais pu refuser ce dénouement heureux à l'enfant et lui infliger, sur un mode en quelque sorte aggravé, la morale pessimiste de La Fontaine. Mais avec ces fables, je cherche à combattre le sentiment d'impuissance politique dans lequel nous baignons, le cynisme diffus qui l'accompagne, le désespoir ou la rage meurtrière qu'il risque de provoquer. Par ailleurs, pour les raisons que je viens d'exposer, je ne voulais pas pour autant trans-

8. On trouvera toutes mes fables sur le site de «Transitions» : <http://www.mouvement-transitions.fr/juste/les-recurrents/helio-milner.html>. Je les écris sous le pseudonyme, pas si transparent que ça, d'Helio Milner. Initialement, je souhaitais que les membres du comité de rédaction puissent s'exprimer à leur sujet sans rien savoir de leur auteur, et aussi tester ce pseudonyme en voyant s'il me découvriraient. Ils ne m’ont pas découverte et ont plébiscité mes trois premières fables. Puis, j'ai continué parce que je me suis trouvée à l'abri dans cette demi-clandestinité. On m'a souvent demandé pourquoi j'avais choisi un nom masculin : je crois qu'il me laisse libre d'habiter les deux sexes. 
former le loup en figure purement vertueuse, comme si de rien n'était, si je puis dire. J'ai donc, dans ma première version du "Loup et l'Agneau", emprunté le "loup rempli d'humanité » du «Loup et les Bergers» et l'ai mis à l'épreuve de sa décision initiale («mourons de faim plutôt»), c'està-dire à l'épreuve de sa rencontre avec l'agneau, tout en suivant d'assez près la fable (c'est-à-dire en introduisant, avec des variations, certains de ses vers les plus célèbres). J'ai transformé aussi l'agneau : le temps que lui laisse le combat intérieur de ce loup-qui-ne-veut-plus-être-un loup lui permet de faire de rapides progrès dans la compréhension de sa situation —et de s'enfuir.

Mais le loup n'étant plus «cruel", comment l'abandonner lui-même à sa mort imminente? Je me suis contentée de conclure sur le commentaire que l'enfant porte sur la réalisation du conteur : «- Pas terrible, observera-t-il. Et maintenant, je m'inquiète pour le loup...»Cette inquiétude finale est très importante : elle signale la complexité du monde souvent occultée par les allégories, et ouvre parallèlement la fable à un rebondissement dramatique. La fable est, en un sens, inachevée. D’où la seconde fable, écrite à la fois par l'enfant et le conteur qui essaient différentes solutions, tout en se coulant encore — mais différemment — dans la fable de La Fontaine :

-Qui t'a permis de, boire à ma source sacrée?

-Aucune source n'est moins sacrée que celle-ci, répondit doucement l'agneau. Tout le monde y vient boire : vaches, renards, enfants... Tu ferais mieux de te passer d'adjectifs si grandiloquents. Songe plutôt à la vie qui coule dans mes veines; ou aux larmes de ma mère et de mon père si tu me manges, comme on m'a dit que tu fais souvent.

- Je suis la Force obscure, reprit le loup en montrant ses babines. La Puissance des Dents. Je suis la Langue, le Gosier, la Fureur...

- Encore ta grandiloquence, dit l'agneau. Ta force et tes dents, je les vois bien, et j'ai très peur de toi. Mais résiste quelques minutes encore. J'ai une solution pour toi. Les bergers ont construit, derrière la bergerie, un élevage de clones, des clones bêtes et insensibles, et incapables, je te l'assure, de reconnaître un loup. Regarde là-bas, vers la ferme : tu trouveras, accrochés à un fil, les habits propres d'un berger. Si tu les enfiles correctement, il te sera facile de t'introduire dans le hangar des clones, où tu trouveras une bonne dizaine d'agneaux fraîchement produits qui ont déjà l'habitude de se laisser manipuler en tout sens...

Le sens moral de ces déplacements va être discuté, discussion qui donne lieu à d'autres propositions.

Mes fables sont en effet essentiellement dialogiques. Et ceci, dès la première. Ce n'est pas à La Fontaine que j'en ai emprunté le sujet, et je n'y raconte rien à proprement parler (en ce sens, c'est à peine une fable). Elle 
interroge la signification de la couleur de la barbe de Barbe bleue - ce qui signifie que les lecteurs sont supposés connaître le conte de Perrault :

Le monde est trop confus pour une fable.

Mais j'invente un enfant à qui lire demain le conte de Barbe bleue, ne manquant pas de lui avouer à la fin que je n’ai jamais, jamais compris pourquoi sa barbe était bleue.

—Essayons d'autres couleurs, proposerai-je.

«Une barbe rousse comme un coq ou la queue d'un renard, comme le bouc d'un démon, lui conviendrait bien mieux".

Nous penserons alors au père de son ami Harold, roux des pieds à la tête, qui pêche le saumon argenté en Écosse, et nous rirons longtemps à l'imaginer en Barbe bleue, un grand coutelas à la main au pied d'un escalier criant : «Descendras-tu que je t'étripe?"

Nous passerons en revue toutes les barbes possibles.

La fable progresse par le jeu des questions-réponses entre le conteur et l'enfant, ce dernier faisant des propositions de couleurs alternatives toutes repoussées par le conteur en raison de leur inadéquation symbolique au personnage, si bien que le conteur conclut finalement à la mystérieuse nécessité de ce bleu.

Aucune de mes fables suivantes n'est racontée hors de ce questionnement. Que les dénouements soient heureux ou malheureux, ils sont toujours subsumés par l'hypothèse anti-tragique qu'il pourrait (ou devrait) en être autrement. Après "Barbe bleue», j'ai (ré)écrit "La Cigale et la Fourmi" et un récit mettant en scène un adage d'Érasme. Un conte, une fable, un adage : je n'ai finalement pas beaucoup exploré les adages (deux autres au total : "Le roi dit "Nous voulons" ", et "Cueille le jour»), ni repris beaucoup de fictions du type de celles des contes : une "métamorphose» à Ovide ("Le Rossignol et l'Hirondelle»), et une "Chèvre de M. Seguin». À la vérité, je crois que les fables que j'emprunte à La Fontaine se transforment quelque peu en contes; et que pour celles que j'invente totalement, elles se situent précisément entre ces deux genres.

Au début, j'avais choisi d'ouvrir mes fables par une formule évoquant la confusion du monde, formule que j'ai variée dans les fables suivantes avant de l'abandonner au profit d'un paysage né peu à peu au fil de mon écriture, dont le premier élément se trouve dès ma quatrième fable, "Corbeau et Renard" :

Le monde, le monde n'est pas trop confus pour une fable!

L'enfant que j'aime y prend goût.

Il devient partie de mon regard.

Le vent pousse une voile sur la mer

Je la suis de mes yeux attentifs. 
Dans mon esprit, la formule «Le monde, le monde (n')est (pas) trop confus pour une fable» devait remplir la fonction de la formule rituelle «Il était une fois", mais adaptée à notre époque, laquelle ne se trouve plus reliée à la stabilité d'un sens venu de jadis. Je voulais mettre en scène le geste et le temps de la transmission, c'est-à-dire souligner combien la transmission était devenue problématique aujourd'hui, souligner le pari que mes fables faisaient d'en renouveler l'essai : un essai de transmission perplexe, troublé et passionné en même temps, parfois désespéré mais le plus souvent joyeux et toujours aimant.

Pour la même raison, j'ai choisi ce destinataire enfant, et le temps futur - parce que notre époque ne parvient plus à se projeter dans l'avenir, à se le représenter :

Le monde est trop confus pour un adage

Mais demain je trouverai l'enfant à qui lire la page

Des loups aux sombres yeux et capables

De paralyser la langue

De ceux qu'ils voient les premiers. («Les yeux des loups»)

Cependant, je me suis très vite rendu compte qu'écrire toujours au futur serait monotone et artificiel. Alors, je me suis mise à mélanger les temps, et j'ai même consacré une fable à cet aspect de mon écriture en prêtant à l'enfant un étonnement à cet égard :

N'en doutez pas, n'en doutez pas, le monde n'est pas trop vieux pour quelques fables!

Qu'il pleuve ou vente, qu'il fasse soleil avec grand ciel bleu ou bien nuées coureuses de nuages, l'enfant arrive à l'improviste certaines de mes après-midi très solitaires qu'il choisit au gré de son âge et des saisons.

Voyez qu'il arrive et entre, j'entends son pas courir sur le chemin de la lande.

- Pourquoi, me dira-t-il, joyeux et jetant son bonnet sur la bergère, pourquoi mélanges-tu toujours tous les temps?

Je lèverai les yeux de ma tâche, étonné :

— Voilà qui est très étonnant! Comment le sais-tu? lui demanderai-je.

Et, croyez-moi, ce n'était pas du vent.

Mais son sourire est très espiègle.

— Je le sais, voilà tout. Et je veux que tu me répondes!

— Le roi dit «nous voulons»...

- Et après?

— Après? C'est très simple. Puisque le roi dit «nous voulons», personne ne peut dire "je veux» et commander plus qu'un roi.

- Je suis plus que le roi, dit-il en bombant le torse. Je suis l'enfant de tes fables.

- Et moi, ton conteur, murmuré-je plus tranquille. J'ai tous les droits sur tous les temps de mes fables.

Et ma voix sera douce comme du miel. 
Outre l'espèce d'instabilité quasi maritime que ces mélanges impriment à la représentation (du moins est-ce l'effet qu'ils me font, l'effet que je vise), je cherche ainsi à traduire, et à contourner ou réparer, la détresse causée par ce cas très particulier qui est le nôtre :

- Je mélange les temps parce qu'ils se sont terriblement mélangés sans qu’on y prenne garde. Je suis comme le maître du temps, j'essaie d'empêcher les grumeaux. Les fables que je te conte sont très anciennes. Je leur tourne la tête vers l'avenir en écoutant le lion rugir?.

—Voilà tout! s'exclame-t-il en se rendormant. («Le roi dit "Nous voulons"»)

François Hartog qualifie le régime d'historicité qui nous caractérise de "présentisme». Il succède à la croyance dans le Progrès et, plus généralement, dans une intelligibilité de l'Histoire : nous n'avons plus d'imaginaire du changement historique, sinon par les vieilles figures du déclin ou de la catastrophe. La figure du déclin, sinistre politiquement parlant, n'apparaît jamais dans mes fables. En revanche, elles se battent avec (et contre) la catastrophe, qui est l'angoisse du conteur et sa tentation dramatique constante, et que l'enfant veut au contraire sans cesse repousser : l'enfant veut l'avenir, c'est-à-dire le possible et l'action.

Car, d'emblée, je me suis donné ces deux personnages de la fictioncadre, le conteur et l'enfant. La présence d'un enfant me permet précisément de scander le temps ou de l'espacer pour le sortir de son présentisme, de figurer la transmission d'expérience sans lui donner un caractère d'évidence. L'enfant a en effet ses propres idées sur le monde, il a lui aussi une forme d'expérience qui est celle de l'attente et de l'espoir. L'expérience du conteur compose avec elle : la mémoire de l'un et l'oubli de l'autre (son innocence, son ignorance) se conjuguent et se rejoignent dans une même curiosité ardente. Leur discussion, souvent animée, me permet de radicaliser toutes les questions et d'interdire l'assertion morale, la sentence. Quoiqu'elles mobilisent quelques «universaux» (le mal et la douleur extrême existent, le désir de justice aussi, etc.), mes fables ne reposent pas sur l'idée de l'universalité de la nature humaine, mais sur la présentation de situations qui, bien qu'emblématiques, conservent tous les traits de la singularité.

En fait, quoique reprenant la tradition allégorique, je ne voulais pas tenir la place d'un fabuliste souverain (d'un narrateur omniscient). En un sens, mon jeu avec La Fontaine laisse à ce dernier toute son autorité : ce

9. La fable passe par le détour d'un rêve de l'enfant comportant un lion amoureux et sa rencontre avec un mystérieux «maître du temps». 
n'est pas avec elle que je rivalise, mais avec son "charme» (ce charme qui rend l'âme "captive» et "attentive», selon ses propres mots $\left.{ }^{10}\right)$, c'est-à-dire avec son imaginaire et son écriture; et je n'ai pu, je ne peux le faire qu'en me plaçant, avec l'enfant, en position d'écoute - d'écoute vibrante, si l'on veut bien m'autoriser cet adjectif, une écoute qui, du coup, est encore là quand j'invente une fable. Quand j'écris, l'émulation avec mon modèle est capitale : ce modèle ne doit pas être écrasant — c'est à cette condition que je peux rivaliser avec lui, c'est-à-dire ne pas être «indigne» de lui. C'est un défi particulièrement vertigineux, particulièrement excitant, lorsque, dans le cas des fables très connues, je joue au plus près de leur lettre tout en la transportant, si je puis dire, dans le monde de ma propre fable de la façon la plus intense possible. Quand je la mobilise, j'essaie de mettre la lettre du texte lafontainien au service de mes propres questions sans rien perdre de son énergie de départ. Ou même parfois, en la rechargeant d'une intensité de premier degré qu'elle peut ne pas présenter dans la fable-source, comme dans «Les Animaux malades de la peste» où je capture le sentiment tragique de terreur en en négligeant la charge parodique (et satirique). Car en actualisant l'horizon des références politiques, je donne au mot "terreur" non seulement un sens émotionnel tragique, mais encore le sens quasi référentiel qu'il a pris depuis la Terreur, et plus récemment le terrorisme, et $j$ 'inverse le rapport figural entre la peste et la terreur : la peste devient la métaphore de la "terreur», elle-même à la fois suscitée par les actions des terroristes et par la politique qui l'instrumentalise :

Cela commence par la terreur.

Tu entends? La terreur.

On ne sait pas d'où c'est venu.

Les uns accusent les dieux, les autres, les hommes.

Ceux qui accusent les hommes disent que les dieux les punissent.

D'autres soupçonnent les puissants d'être la cause de tout ça.

Mais ils sont pris aussi dans la terreur.

D'ailleurs, même les plus puissants commencent à avoir peur.

Mais ils ne sont pas décidés pour autant à renoncer à leur puissance.

—J'ai peur, dira l'enfant sourdement.

— Moi aussi! La terreur, ça ne fait rire personne.

Tout le monde est touché même si tout le monde ne meurt pas.

Et beaucoup meurent.

IO. «C'est proprement un charme : il rend l'âme attentive, / Ou plutôt il la tient captive, / Nous attachant à des récits / Qui mènent à son gré les cœurs et les esprits." (La Fontaine, "À Madame de Montespan», Fables, en tête du «Second recueil», v. 7-IO) 
On ne connaît pas cette maladie, on l'appelle peste faute de mieux.

On ne connaît pas ceux qui, parfois, entrent dans des lieux pour ajouter les morts aux morts.

On ne connaît pas ceux qui laissent derrière eux des corps déchiquetés.

Parfois même, il semble que ce ne soit personne.

Et plus personne, entends-tu, plus personne ne se sent en sécurité.

Après avoir confié au conteur l'évocation de cette "peste», après avoir laissé la «terreur» s'installer entre lui et l'enfant, j'ai continué en serrant de plus près le texte lafontainien :

L'enfant regarde la terreur dans mes yeux, et je vois comme elle grandit dans les siens.

-J'ai peur, dira-t-il encore.

— Moi aussi! Moi aussi, dirai-je sourdement.

Chez les animaux ça va tout aussi mal.

Les tourterelles se fuient.

Les fauves n'aspirent plus à leurs proies,

Et pourtant les proies meurent elles aussi.

Partout se chuchote qu'il faut tuer le roi,

Et puis les magistrats, et puis les médecins,

Les militaires, la police,

Et les malades bien sûr,

Et tout refaire à zéro.

Et cela ferait encore plus de morts.

Pressé par ce désastre, le lion tient conseil.

Mes chers amis, j'ai décrété l'état d'urgence comme il se doit.

Cela nous a causé quelques morts en plus, et le peuple murmure.

Les dieux, n'en doutons pas, demandent quelque grand sacrifice.

Il faut que le plus coupable d'entre nous périsse.

Que chacun s'accuse donc de bonne foi : la faute impardonnable de l'un de nous est certainement la cause cachée de la colère du Ciel.

Tout le monde sait que je suis le roi Lion. L’armée m’obéit. Les banques m’obéissent. Les prêtres m’obéissent. Mais je ne suis pas sans défaut. Par exemple, j'ai parfois abusé de mon pouvoir et fait tuer force moutons, beaucoup plus de moutons que je ne pouvais en manger. À la vérité, j’ai même parfois ajouté les bergers. Ils ne m'avaient rien fait, c'est vrai : c'était un pur désir de goûter aux bergers aussi.»

\section{Il y a des moments plus légers :}

Son lait bien chargé sur la remorque de son vélo,

Notre laitière roulait avec entrain,

L'air étant vif, le chemin long.

Dans sa tête tout en roulant,

Elle refait le compte de ses bidons,

Les traduit en argent trébuchant,

Songe à tout ce qu'elle va se procurer : 
Des poussins par exemple, tout jaunes et piaillant, Qu'elle appelle soir et matin en leur versant du blé.

Pas difficile qu'ils deviennent poulets et chapons gras à souhait!

Elle en tue quelques-uns qu'elle rôtit pour les vendre,

Fait couver aux poules des poussins en foule,

Bref en tire tout ce qu'il faut pour acheter un ou deux cochons déjà rondelets, Que je gave avec du son!

Les revendre, quoi de plus simple!

Et pour acheter quoi? Une vache et son veau

Qui bondiront au milieu de l'herbe folle! ("La laitière et le pot au lait»)

Parfois, ma récriture est plus distante à l'égard de mon modèle :

Un jour l'écrevisse dit à sa fille : «Mais regardez donc comment vous marchez! Est-ce que vous ne pourriez pas aller droit?»

— Et comment voulez-vous que je fasse? répliqua la fille. Avez-vous vu comment vous marchez vous-même? Pourquoi marcherais-je droit dans une famille où chacun va tordu?

L'enfant et moi, nous nous sourions, contents.

Il attend.

Je me tais.

—Et puis? demande-t-il, étonné soudain par mon silence.

- C'est fini, répondrai-je.

— Fini! Je ne te crois pas!

— Mais si, je t'assure. Il faut maintenant penser à la morale...

— La morale! Mais non, je ne te crois pas! Il n'y a même pas eu d'histoire, et tu veux une morale? Ça n'a pas de sens!

—Enfant de mon cœur et de mon imagination, les choses bien souvent n'ont aucun sens.

Dans ma mémoire passent des bousculades brutales où des hommes projetés s'hébètent sans rien comprendre. Et des poings levés et des bras qui essaient de se protéger et des détresses galopantes propagées dans les regards de visage en visage. Dehors le vent se lève, il hérisse méchamment la crête des vagues.

- Je l'entends, déclare l'enfant qui ne me quitte pas des yeux. Et si je ne l'entendais pas, je le reconnaîtrai dans tes yeux. Il va se calmer et les vagues aussi, et je me demande souvent pourquoi ce noir tourment. Mais quand même, nous n'en sommes pas à la morale. Ta fable n'est pas finie.

Alors, alors, écoutant le vent, écoutant le temps, je céderai à sa colère belle.

- Tu veux que je fasse réagir la mère?

— Par exemple, dit l'enfant, royal, en se réasseyant dans ma bergère. («L'Écrevisse et sa fille»)

Mes fables doivent causer à l'enfant une émotion forte, le bouleverser, l'inviter à parler, à intervenir. C'est sous cette condition d'une émotion à la fois partagée et disputée que j'écris et que je «cite» La Fontaine : la scène de partage entre le conteur et l'enfant constitue une sorte de scène- 
témoin : elle me surveille (ou me bienveille) pour que le plaisir procuré par ma fable soit isomorphe au plaisir procuré par le conteur à l'enfant (et réciproquement), un plaisir qui se souvient de mon propre plaisir à lire les fables de La Fontaine (et quelques autres textes ${ }^{\mathrm{II}}$ ).

La relation entre le conteur et l'enfant s'est enrichie et complexifiée au fil des fables au point de devenir elle-même porteuse de sens. Leurs deux figures commencent à prendre une consistance qui pourrait déboucher sur du romanesque (si cela advient, la fable morale se suspendra). Les tensions entre eux, la polarité qu'ils incarnent de façon souple, ont aussi une valeur allégorique. En gros, je réserve au conteur une expérience qui comprend la mémoire des échecs amoureux et politiques, des traumas historiques; et à l'enfant, la révolte, l'espoir que le monde peut être sinon racheté, du moins réformé (voire révolutionné). La plupart du temps, l'enfant parvient à infléchir le dénouement de la fable, à la détourner d'une fin malheureuse. Mais je ménage des rebondissements, pour deux raisons : il ne faut pas que le lecteur sache par avance ce qu'il en adviendra (c'est le principe esthétique de la variation); je veux maintenir présente l'existence du conflit lui-même et incertaine la solution (c'est mon souci éthico-politique). Il m'arrive même de ne pas suivre La Fontaine dans ses dénouements "heureux» : ma récriture de la fable "Le Savetier et le Financier» substitue un pêcheur au savetier — et le financier est notre contemporain, un trader. $\mathrm{Si}$, comme dans cette fable, on met la pauvreté en relation avec la richesse, je ne peux pas l'associer aujourd'hui à l'insouciance ni même à la sagesse - sauf dans "Le Rat des villes et le Rat des champs", où ce dernier présente une forme de sagesse vigilante qui pourrait être celle d'un «zadiste» de Notre-Dame-des-Landes. Dans «Le Savetier et le Financier», la figure du pêcheur a fait surgir les «boat-people»:

- Il arrive que le pêcheur, dirai-je le cœur serré, trouve des corps morts dans la mer. Beaucoup, beaucoup de corps morts. Pendant ce temps, le trader regarde le cours des actions monter, baisser, il en est au pétrole...

- C'est ça, ta fable? me dira l'enfant, rageur.

- C’est ça, ma fable, répondrai-je, le cœur serré...

- Je refuse, criera l'enfant. Ça ne marche pas comme ça! Il y a plein de gens autour du pêcheur. Toi, moi, beaucoup d'autres. Ça fait une chaîne de gens! Ça fait une chaîne de possibilités possibles!

— Tu ne peux pas faire taire le vent comme ça, murmurerai-je doucement.

II. C'est ainsi que la lecture d'Elio Vittorini, Conversation en Sicile (1948) — le dialogue entre la mère et le narrateur tout particulièrement - a nourri l'écriture de mes fables à mon insu. 
Ici, ma fable tourne à la tragédie sans solution politique dans le récit, contrairement aux «Animaux malades de la peste» où l'enfant invente un dénouement révolutionnaire qui sauve l'âne.

À travers ce couple du conteur et de l'enfant, je dialogue avec ma mémoire (mes engagements politiques de jeunesse, les espoirs effondrés, etc.) et avec la jeunesse qui veut, tout de suite, un changement radical de société. Mais le conteur n'est pas moi. Lui et l'enfant sont aussi des "possibles» de chacun (donc de moi également). Entre eux deux, le conflit est joueur plus qu'agonistique. Le conteur consent à la jeunesse rebelle, indignée, joyeuse de l'enfant, à sa joie triomphale comme à sa colère; quant à l'enfant, non seulement il consent au conteur, à sa mélancolie, à son savoir déceptif, mais il s'inquiète de lui parfois. Entre eux deux, il y a de l'amour, la vie circule, et la sagesse prudentielle que la forme-dialogue fait naître est ainsi nourrie d'élans et de désirs. La symbolisation du paysage, peu à peu surgie au fil de mon écriture des fables successives, y compte pour beaucoup. Le conteur habite une maison au bord d'une falaise. De la pièce où il se tient, on voit le ciel, la mer, le vent. L'enfant arrive par la lande et après être entré, il s'installe dans une bergère pour écouter. Récemment, un jardin a surgi — et la relation entre le conteur et l'enfant a gagné en quotidienneté :

Il sort le premier en sautillant. Ses bras écartés font un mouvement d'oiseau.

Il prend le chemin qui court et tourne au coin du mur.

C'est un virage de mouette, d'albatros ou de goéland.

-Où allons-nous?

- Au jardin.

Parfois, le jardin est pris de givre. L'herbe brille, les buis scintillent au soleil.

Parfois, il y a même des roses écloses en hiver.

Il s'arrêtera. Je vois ses cils, ses yeux pensifs.

Je m'arrête aussi pour penser.

Il s'est arrêté, on le croirait face à une tombe. Mais ses joues sont vives, et vives ses lèvres, vive son attention.

—Elles sont fanées, murmure-t-il.

Je les ai vues aussi.

—C'est normal, murmuré-je. Les boutons s'ouvrent, les pétales se déploient, le cour s'épanouit. Et puis les fleurs se fanent.

Il se tourne vers moi, il me tire la langue. Je la lui tire aussi.

Il fait des grimaces et rit aux éclats. Je ris aussi.

Il repart en courant et rentre dans la maison.

- Je veux un chocolat chaud, dira-t-il.

Nous savourons la boisson en silence. L'odeur suave nous relie, elle donne couleur au silence. 
Lorsque je commence une fable, mon humeur du moment donne sa couleur au paysage, qui est une sorte de visage - et l'enfant lit souvent dans les pensées du conteur et entre en dialogue direct avec elles grâce à cette projection de l'humeur du conteur sur le paysage. Mes fables se situent en effet explicitement aux frontières du rêve et du vraisemblable : la maison au bord de la falaise où habite le conteur, le temps qu'il fait dehors avec la rumeur des vagues, la bergère dedans où l'enfant s'installe, tout ceci pourrait avoir une consistance analogue aux maisons de l'enfance stylisées par le souvenir qu'on en garde. Mais ce décor a pour moi une puissance onirique, et l'on ne sait jamais d'où arrive l'enfant (en tout cas, jusque-là), il raconte seulement parfois ce qu'il a vu en traversant la lande (des animaux, qui engendrent la fable). En un sens, le conteur est celui qui menace le rêve de virer au cauchemar, tandis que l'enfant veille au contraire sur le désir et l'espoir qui en sont le moteur caché.

Si je devais faire une sorte de typologie des fables que j'écris, je dirais qu'elles mettent en scène, en gros, deux sortes de dilemmes : ceux que je pourrais qualifier d'affectifs (qu'en est-il de l'amour, du désir, qu'est-ce qu'ils peuvent? cela engage une écriture que je dirais elle-même érotisée, c'est la plus heureuse); et des dilemmes politiques. Entre les deux pôles, je rencontre le crime, la mort («Le Rossignol et l'Hirondelle», «La Mort et le Mourant»).

Pour l'instant, j'écarte les fables «sociales» (du type «Les Deux Mulets»). Je ne me sens pas du tout attirée par la satire : je ne cherche pas à écrire une «comédie humaine». Les «caractères» non plus ne m’intéressent pas. Nos vies ne reposent plus sur la lecture juste des types humains, sans doute parce que nous ne dépendons plus directement, immédiatement, les uns des autres : nous dépendons d'institutions aux rouages complexes, il y a des contrats, du droit — ou de la bureaucratie la plupart du temps sans visage, en tout cas sans visage familier... Et si nos psychismes ont plutôt gagné en importance depuis l'époque de La Fontaine, ce sont leurs méandres qui nous intéressent — ces méandres que mes fables évoquent à travers les paysages, et parfois un bref monologue intérieur du conteur.

Je conclurai en évoquant le renard. Sauf dans "Les Animaux malades de la peste», il n'a pas réapparu depuis "Corbeau et Renard", où je ne lui donne pas le beau rôle : c'est le Corbeau qui le confond. Je vois deux raisons à cet évitement. La première, c'est que le renard risque trop d'illustrer le cynisme actuel, et je ne veux pas en faire l'éloge. La seconde est plus joyeuse. Sur son versant positif, le renard, avec ses inventions, constitue une sorte d'alter ego du fabuliste. Or, c'est à l'enfant que je confie ce rôle. Non seulement il possède, avec ses demandes, une puissance de rebon- 
dissement analogue à la fonction dramatique du renard, mais il arrive qu'il intervienne directement, comme par ruse, pour voler au conteur le dénouement attendu.

Il n'est pas du tout certain qu'il ait raison: mais sans ses gestes, le goût du monde se perdrait, et l'injustice serait assurée de régner jusqu'à la fin des temps. Voici l'horizon moral qui est le mien. 